\title{
The unsuspected intrinsic property of melanin to transform light energy into chemical energy and the Warburg effect: Connotations in cancer biochemistry
}

\author{
Arturo Solis Herrera* \\ Human Photosynthesis ${ }^{\circledR}$ Research Center, Aguascalientes, México
}

\begin{abstract}
Otto Warburg found, in 1924; that unlike most normal tissues, cancer cells tend to ferment glucose into lactate even in the presence of sufficient oxygen to support mitochondrial oxidative phosphorylation. Aerobic glycolysis is a poorly efficient metabolic pathway to generate ATP, thereby the advantage it confers to cancer cells remain unexplained. Reactive medicine is failing markedly in all countries. This is expected due is a kind of medicine based mainly in around 7000 intracellular chemical reactions already described in scientific literature, however only 199 are described in the same way in the different sources of information, and there are significant controversies, in the 6801 remaining, as they are not described in the same manner in the different available databases. This current lack of understanding of cell biology is congruous with reactive medicine results. Less of $3 \%$ of main diseases are resolved by institutional medical systems; the $97 \%$ are just palliated. Our finding of intrinsic property of melanin to transform light energy into chemical energy by means of water dissociation, like chlorophyll in plants; will mark a before and after in the field of cell biology and thereby in the practice of medicine. Therefore, the sacred role of glucose as source of energy by excellence of cell now is broken into small pieces. Glucose is just carbon chains source to our body but not energy source in anyway.
\end{abstract}

\section{Introduction}

Since 1924 has been detected that cancer cell metabolizes glucose in a manner that is distinct from normal cells. It seems that fermentation of glucose to ethanol might apply to mammalian tissues. Almost 100 years later, a definitive explanation for Warburg's observation has remained elusive. In the presence of oxygen, most differentiated cells primarily metabolize glucose to carbon dioxide by oxidation of glycolytic pyruvate in the mitochondrial tricarboxylic acid cycle, with minimal lactate production. It is only under anaerobic conditions that normal or differentiated cells produce large amounts of lactate. Oppositely, most cancer cells, produce large amounts of lactate regardless of the availability of oxygen and hence their metabolism is often referred to as "aerobic glycolysis".

AT first, mitochondria defect in cancer cells was proposed; however, subsequent work showed that mitochondrial function is not impaired in most cancer cells. The metabolism of glucose to lactate generates only two ATPs per molecule of glucose, whereas oxidative phosphorylation generates up to 36 ATPs upon complete oxidation of one glucose molecule [1].

Throughout all these years, it has tried to explain the Warburg effect, whereas the ATP as the energy source par excellence of the cell, and despite best efforts by various researchers, was is able to secure a satisfactory explanation, or that at least lead to some treatment preventive, palliative, or much less corrective in cancer patients.

The purpose of this work is examine the possibility of explaining the Warburg effect, but whereas the ATP as a molecule whose primary function is to control the levels of phosphate, thermodynamically unstable but kinetically stable molecules; and proposing a different role for mitochondria, probably as an organelle which increases the temperature and its counterpart, the ATP, which in turn generates as in our experiments, the transformation of ATP into ADP decreases the temperature.

The function thermogenic that we propose for the mitochondria is based on the observation that organisms which originated in cold periods of the history of the Earth, tend to have more mitochondria than organisms which originated in relatively warm times. Observation reached human bodies, as fair-skinned people; reach up to $83 \%$ more mitochondria than dark-skinned people.

And in relation to which the mitochondria produces the same counterparty, ATP; is somewhat not unusual in nature, and we can cite the example of the pancreas, which produces insulin, a hormone that, among other effects; it lower blood glucose levels, but it is the same pancreas that produces glucagon, a hormone that tends to raise blood sugar levels. That is, the mitochondria, one of smaller organelles; raises the temperature, and the ATP decreases it to become ADP.

\section{The metabolic needs of proliferating cells}

To produce two viable daughter cells at mitosis, a proliferating cell must replicate all of its cellular contents. This imposes a large requirement for building-blocks, i.e. nucleotides, amino acids, lipids, and thereby glucose as main source of carbon chains. In other words, glucose is used to generate biomass, but energy, defined as anything

Correspondence to: Arturo Solis Herrera, Human Photosynthesis ${ }^{\circledR}$ Research Center, Aguascalientes, México; E-mail: comagua2000@yahoo.com

Key words: melanin, energy, glucose, warburg's effect, hydrogez

Received: September 20, 2015; Accepted: October 08, 2015; Published: October 12,2015 
that produces a change, is taken from water, like plants.

\section{Melanin, the human chlorophyll}

The unsuspected intrinsic property of melanin to absorb the full electromagnetic spectrum, visible and invisible; dissipating absorbed energy in non-radiative manner through the dissociation of water molecule, like chlorophyll in plants; implies a new paradigm in cell biology, because signify that our body is able to take energy from water, like plants [2]. Before our crucial observation, the only known molecule able to do something like this was chlorophyll, and it was unique.

Chlorophyll absorbs extremes of visible light, and with that energy is able to dissociate the water molecule in an irreversible form, into its gaseous components: Hydrogen and Oxygen. The reaction can be described in the next way:

$$
2 \mathrm{H}_{2} \mathrm{O}_{\text {(liquid) }} \rightarrow 2 \mathrm{H}_{2 \text { (gas) }}+\mathrm{O}_{2 \text { (gas) }}
$$

The real value product is molecular Hydrogen, because is the energy carrier by excellence in the universe, therefore our body cannot be different in anyway. By other side Oxygen is toxic at any concentration; thereby the plant expelled it to atmosphere. Water dissociation is a very expensive reaction from energetic point of view, its replication in laboratory requires $2000^{\circ} \mathrm{C}$. However, chlorophyll is able to do it at room temperature. These are some reasons why was considered a unique reaction, besides to be considered as the most important chemical reaction in the world due is the first step in food chain.

Transformation of light energy into chemical energy is reached through a sequence of chemical reactions whose main final product is glucose. These glucose molecules are the building blocks of plant cell constituents, as walls, organs, $\mathrm{pH}$ regulation, ATP; etc. Approximately $99 \%$ of biomolecules have carbon atoms coming from glucose. The mechanics of thought was that chlorophyll would transform light energy into chemical energy and storing it in the links of glucose. Thereby glucose molecule itself requires energy to form and also to maintain shape, especially when it is immersed in water. That is, chemical energy should exist prior to the appearance of glucose, and this since the beginning of time and until today. We could say that the origin of life on Earth starts with the highly ordered chemical energy that emanates from the melanin. And that can be written in the following way:

$$
2 \mathrm{H}_{2} \mathrm{O}_{\text {(liquid) }} \leftrightarrow 2 \mathrm{H}_{2 \text { (gas) }}+\mathrm{O}_{2 \text { (gas) }}+4 \mathrm{e}^{-}
$$

This process of dissociation and renovated water, occurs within the melanin with surprisingly exact precision. When melanin and water are within the limits that we might consider normal, i.e.: when the reactants are its purest form possible, hydroxyl radicals will not form, will not form superoxide anion or not Ionic hydrogen will be generated. When melanin works in optimal conditions their products are, invariably; molecular hydrogen $\left(\mathrm{H}_{2(\text { gas })}\right)$, diatomic oxygen $\left(\mathrm{O}_{2(\text { gas })}\right)$ and 4 electrons of high energy for every two molecules of water reformed.

\section{This is:}

$$
2 \mathrm{H}_{2} \mathrm{O}_{\text {(liquid) }} \leftrightarrow 2 \mathrm{H}_{2 \text { (gas) }}+\mathrm{O}_{2 \text { (gas) }}+4 \mathrm{e}^{-}
$$

And takes place with amazing accuracy and constancy, always and when the reactants, melanin and water; retain their basic physicochemical properties. Deviations occur when melanin is damaged e.g. when iron is combined with the melanin, and then the Nano metric precision is lost in greater or lesser degree, depending on the degree of contamination; and they begin to appear hydroxyl radicals, superoxide anions; e hydrogen ion; which distort the finely tuned processes of generation and distribution of energy that nature has patiently developed over four billion years of evolution.

The same happens with water, which, when is it contaminated one way or another, for example with metals, pesticides, herbicides, with industrial waste; anesthetic agents, It begins to change, and the first thing you lose is viscosity and then other properties, with what the reaction is no longer accurate, begins to occur a bias, and something is going to happen with the generation and distribution of energy, which, from the clinical point of view is unpredictable, given that involved confounding factors such as age, weight, height, sex, previous state of health; etc. also play a role.

We must correct the dogma that the cell begins to get and therefore to use chemical energy after mitochondria synthesize ATP, so far, trying to explain the origin of life and of diseases using as base these metabolic pathways has led us to a pothole, multiple theories in different ways, which are confusing, contradictory; and of course fail when it comes to clinical application.

\section{Melanin, generalities}

Melanin is a molecule that has been traditionally relegated in biochemistry, for different reasons, and only has been given the biological role of a simple sunscreen, which protects us from dangerous UV rays. It is a primary colour agent in hair, skin and eyes. In eyes, retinal epithelium melanin absorbs harmful radiation. Melanin is the darkest substance man ever known, however the main energy deactivation pathway for absorbed radiation was unknown. The role of melanin in dark places as brain stem, inner ear, eye, etc., is poorly understood, and is considered pure speculation. So little is what is known of melanin, that it is postulated that the solar radiation may induce cytotoxic responses inside her.

We find, incidentally; during a descriptive study about the three main causes of blindness in the world, started in 1990 and ended in 2002; the unsuspected intrinsic capacity of melanin to transform light energy, visible and invisible, into chemical energy, through the dissociation of water. Our working hypothesis was on vascular changes in the optic nerve that occur in the three leading causes of blindness in the world, and they are: glaucoma, diabetes and macular degeneration related to age.

The studio was focused at the beginning; in trying to find early alterations in the capillaries of the optic nerve that would allow us early diagnosis and therefore treatment also early. But the optic nerve in humans is very small, usually measure the equivalent of twelve together human hair, so it was necessary to important magnifications that allowed us to observe and digitize the morphological changes of interest to our study (Figure 1-4).

The answer to our question of why the insistence of nature in place melanin in the vicinity of the optic nerve, in all patients, and even in the healthy; It could not be answered in the literature search. Including rarely mentioned; it seems that it is something that happens and nothing else. They are embryonic remnants occasionally said.

But nature just insists on important things, and on the other hand not wasted nothing. So we are still looking for answer and at the same time we were still observing patients, prompting us to detect a second key fact: we detected an inverse correlation between the amount of melanin present and the amount of blood vessels present.

We began to notice that the amount of melanin influences 


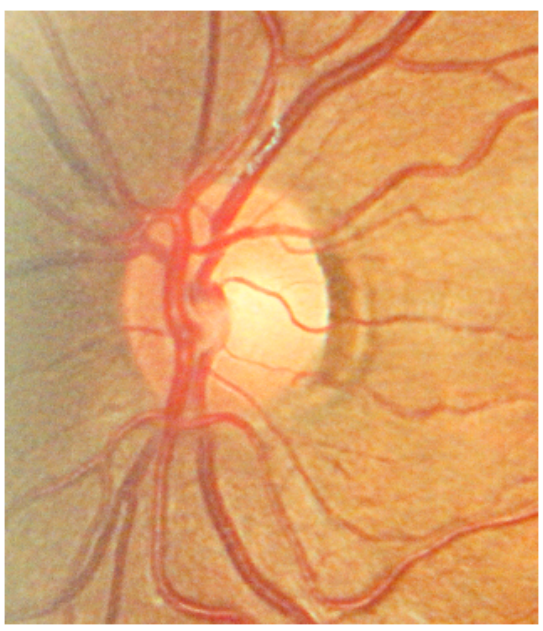

Figure 1. Normal optic nerve of the left eye, the blood vessels were our main variable in study, however the constant presence of melanin in the almost 6000 studied during the period 1990-2002, draw powerfully our attention.

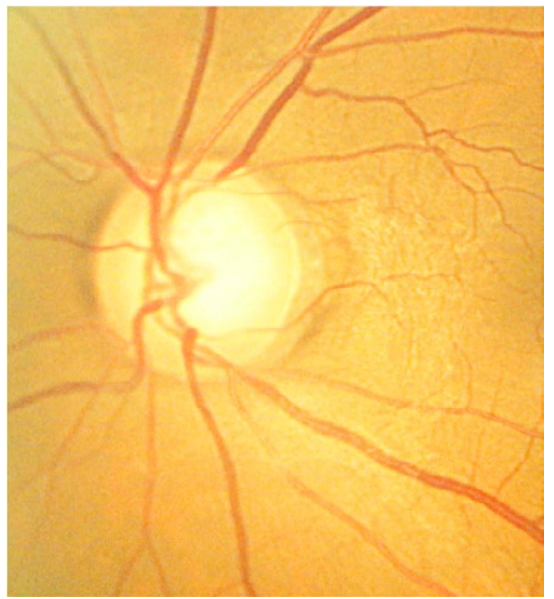

Figure 2. Optic nerve, left eye; the presence of melanin in this case of glaucoma is in nasal side, at the right of the photography, over the maculopapilar bundle, the presence of gliosis. Melanin is always nearby optic nerve, in healthy and sick patients.

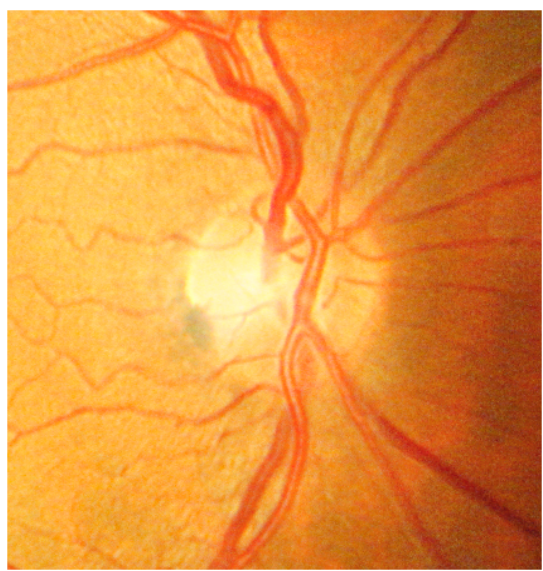

Figure 3. Melanin does not have a uniform or similar distribution around the optic nerve in all people, it is different in every single patient, whether healthy or sick, but always present. powerfully the number of blood vessels present in the area. This is: more melanin less number of blood vessels, and vice versa (Figure 5).

In the right lower part of Figure 5, we can see reddish lines that go in several directions. They are choroidal blood vessels that are not normally visible. Normal vessels from the choroid is closest to the top right of the picture 5, where only appreciated a uniform orange color, and that is given by a mixture of structures that isolated blood vessels become unrecognizable. The number of vessels containing the choroid is such that fade between them. But when the amount and activity of melanin increases, the vessels tend to disappear. It was an observation that was repeated over and over again. There was no doubt, somehow the melanin had a previously unnoticed anti-angiogenic effect.

As it was not described in the literature, we realized the task of finding the answer, but the answer was not simple since it was the melanin which is capable of markedly influencing the size, shape and distribution of the blood vessels and not vice versa. As melanin does not possess enzymatic machinery, either genes or something similar; the factors that produced the vascular endothelium were not the explanation we were looking for, such as VEGF.

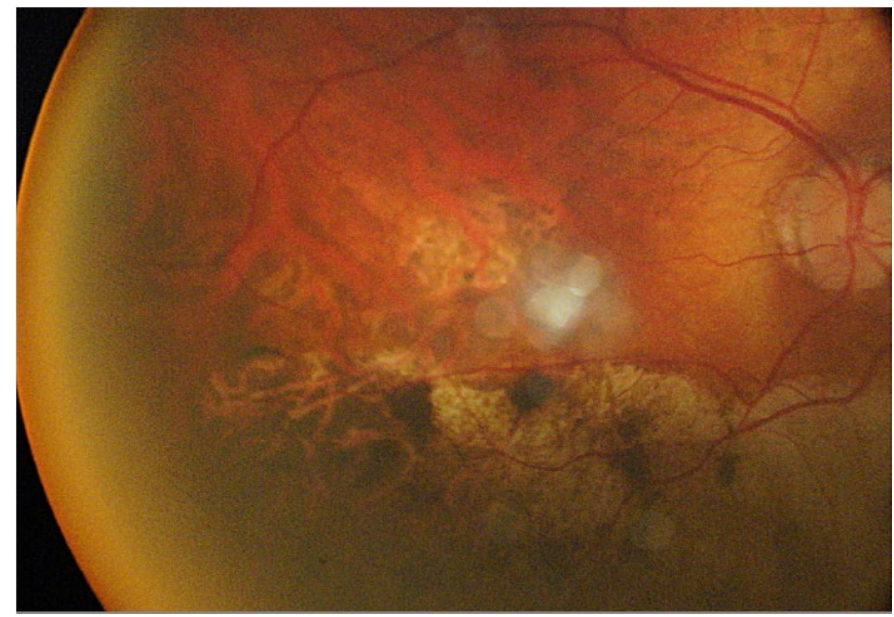

Figure 4. Melanin is always involved in pathological processes of the eye. Until now thought that it was a simple inert actor, which was accumulated, or that simply migrated, or disappear. But now that we understand its enormous importance as primary, fundamental energy source of the cell, thereby, clinical interpretation of photographs like this, changes dramatically.

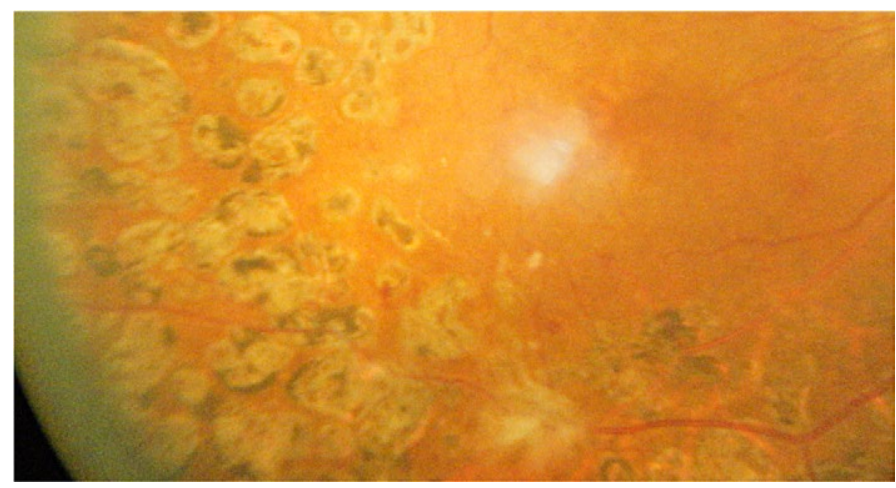

Figure 5. The melanin in the interior of the eye, as well as the skin, is activated by electromagnetic radiation, as in this case of diabetic retinopathy that was treated with laser photocoagulation. The pigment is activated, although not in a uniform manner. When oxygen-sensitive electrodes has been placed so close this scars during intraocular surgeries, i.e. vitrectomy; the level of oxygen is significantly higher in the pigmented areas. 
Herrera AS (2015) The unsuspected intrinsic property of melanin to transform light energy into chemical energy and the Warburg effect: Connotations in cancer biochemistry

Perhaps the factor which initially us enthusiasm was Pigment Epithelium Derived Factor (PEDF) but it was for a short time, given that melanin does not have structures that could be equated to cell membrane receptors. And on the other hand, the effect of melanin is surprisingly consistent, is nowhere near $30 \%$ in average obtained peptides via due to biological variability that significantly decreases its efficiency. Conversely, the melanin antiangiogenic effect reaches more than $90 \%$. So we started to look for some kind of message that did not require receivers. Incidentally, in the same period we began to detect the levels of oxygen in the pigmented tissues were very high in comparison to similar tissues but with least amount of pigment. At least in ocular tissues, the difference reached $34 \%$. So that we began to frantically search for that molecule or molecules in ocular tissues might be able to donate such amount of oxygen and consistently so, without that you will notice therefore is not known any molecule that is able to do so without compromising herself.

We find the answer in February 2002, after thoroughly studying other known options or less possible. The only real possibility was that melanin has the unsuspected intrinsic property of absorbing the visible and invisible light, and that absorbed energy is dissipated separating the water molecule. At the beginning we thought the reaction as well:

$$
2 \mathrm{H}_{2} \mathrm{O}_{\text {(liquid) }} \rightarrow 2 \mathrm{H}_{2 \text { (gas) }}+\mathrm{O}_{2 \text { (gas) }}
$$

Principle think that it should be similar to that occurred in chlorophyll, but as the investigation continued to make progress, experimental observations showed us another amazing fact: the melanin was not only able to dissociate the water molecule, which is the part energetically expensive process, they also possessed the amazing ability to re-shape it, so the equation at the end; It was in the following manner:

$$
2 \mathrm{H}_{2} \mathrm{O}_{\text {(liquid) }} \leftrightarrow 2 \mathrm{H}_{2 \text { (gas) }}+\mathrm{O}_{2 \text { (gas) }}+4 \mathrm{e}
$$

The process is fully reversible in melanin, but not in the chlorophyll (Figure 6-9).

From the first time that we conceive as a possibility real that melanin should be the equivalent of the chlorophyll in humans, we then gave the task of finding experimental and clinical data that could support our discovery. The vast majority of the data that we found

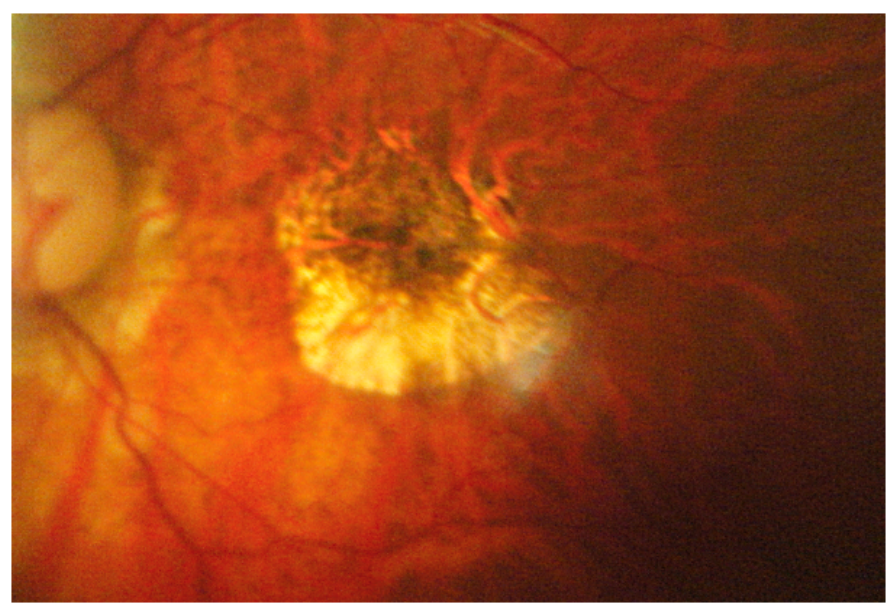

Figure 6. Example of a patient with age related macular degeneration. The Anatomy of the macula is distorted. The pigment has accumulated in a zone and apparently disappeared from another. From here we can see the effect of melanin on the blood vessels. The characteristics of the vessels are significantly different depending on the amount of melanin present.

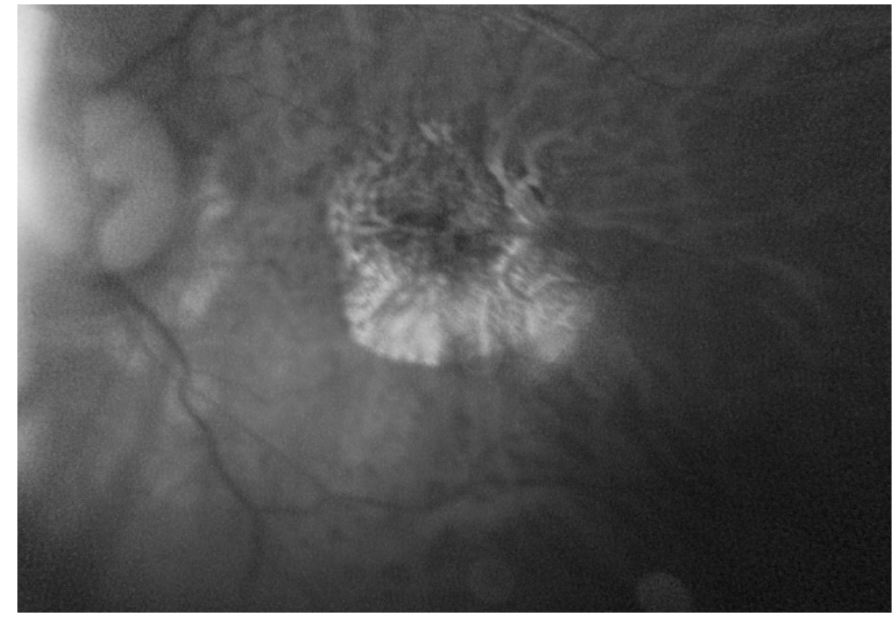

Figure 7. The same patient 6 photography, but in black and white. allowing contrasting some important details in regards blood vessels and melanin.

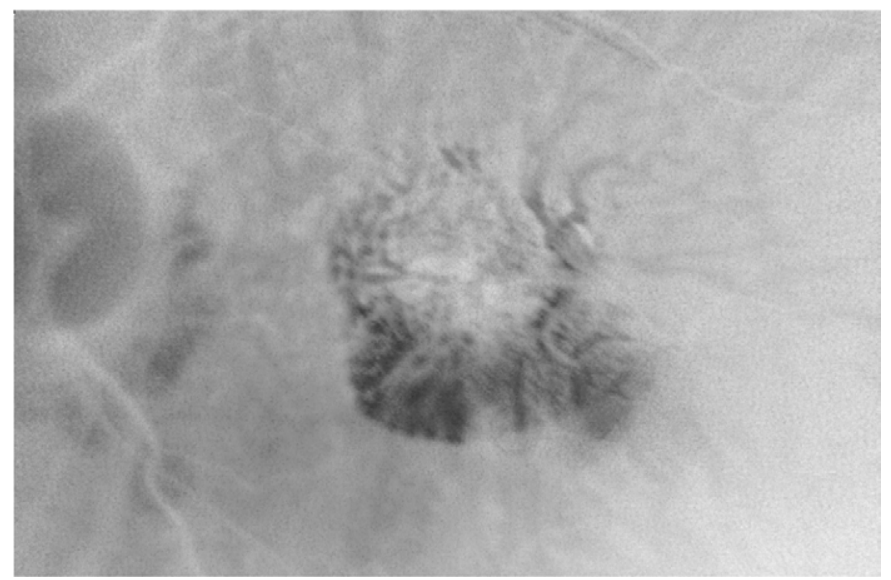

Figure 8. The macula of the patient in Figure 6, but negative. The Anatomy of the vessels differs significantly depending the presence or absence of melanin.

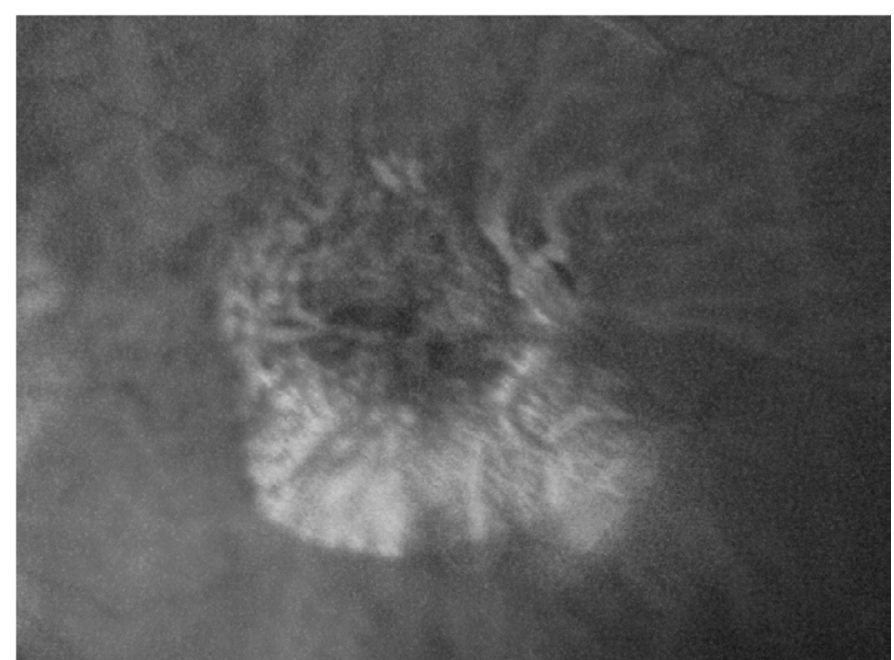

Figure 9. The macula of the patient in Figure 6, but with higher magnification; the structura elements show differences in diameter, size, direction, etc.; between areas with major and minor pigment. There are confusion factors that mask the anti-angiogenic effect of melanin. 
were subtle, indirect, but we expected something like this given the hydrogen gas (molecular) is almost impossible to measure with current techniques, and much less in a living tissue, the more we would have to find their biological effects. Oxygen is much easier to measure, and even there is abundant literature on the subject, although it is based on another dogma, which hemoglobin transports it from the atmosphere to tissues.

In the case of diabetes, Laser photocoagulation is a technique used since the late 1960s, and which consists of the application of intense monochromatic light on the retina, with the intention to form retinal and choroidal scars. Such therapeutic conduct originates in the ancient observation that the presence of scars in the choroid and retina seems to decrease the progression of diabetic retinopathy. But since then and to date there is no explanation.

Choroidal scars are characterized by the presence of melanin, and oxygen levels have been studied in them, although the explanation that was given was very different, because it was said that photocoagulation destroys parts of the outer retina, thus reducing their consumption of oxygen and allowing oxygen to diffuse from the choroid towards the inner retina, which improves the oxygen supply to the inner portion of the retina [3].

In animal laboratory experiments, results were that the measurement of pre-retinal oxygen tension over laser-treated and intact retina in normal and diabetic cats, the oxygen tension was significantly higher over the area given pan-retinal photocoagulation than over the intact retina when the cats breathed $100 \%$ oxygen and the same trend was seen when the cats breathed $21 \%$ oxygen.

This is a very interesting experimental data for us but that was misinterpreted by the authors, however fit very well with our finding. On the one hand it shows that hemoglobin does not have neither the capacity nor the function to transport oxygen from the atmosphere into the tissues, because if I was, the difference in the concentration of oxygen breathing animal laboratory, had changed the tissue levels in a meaningful way, but there is no change, shows that you don't have relationship between the atmospheric oxygen with the concentrations of oxygen in the tissues [4]. The explanation is that hemoglobin is able to dissociate the water molecule in an irreversible manner and produce oxygen and hydrogen, as does the chlorophyll in plants. And on the other hand, the increase in the amount of melanin in the scars of choroid, explain congruously that oxygen levels are significantly higher in areas with scars that the healthy area (Figure 10).

Perhaps one of the factors that have perpetuated the misconception that glucose or ATP is energy sources is our mundane concept that we have to see how the cell consumed 'fuel'. For most mammalian cells in culture, the only two molecules catabolized in appreciable quantities are glucose and glutamine. By other side, cells deficient in ATP often undergo apoptosis. From this perspective, it becomes clear that Glucose, Glutamine and ATP have important biological roles, but they can still be important without much to do with power generation.

\section{Why cancer cells prefer glycolysis, an inefficient meta- bolic pathway?}

\section{The Warburg Effect}

In the 1920s, Otto Warburg first proposed that cancer cells show increased levels of glucose consumption and lactate fermentation even in the presence of ample oxygen. This is the so-called Warburg effect. It is now accepted that glycolysis provides cancer cells with the

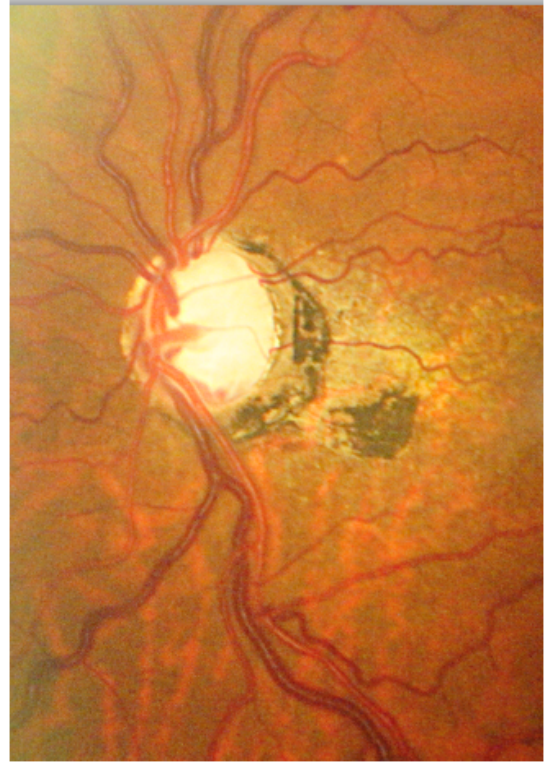

Figure 10. Typical appearance of a scar that involves the retina and the choroid. It can be of many forms, in many locations, the number of vessels is variable, the colour, appearance, etc. But what can never be missing is the presence of melanin.

most abundant extracellular nutrient, glucose, to make ample ATP metabolites intermediates, such as ribose sugars, glycerol and citrate, non-essential amino acids, and the oxidative pentose pathway, which serve as building blocks for cancer cells.

Mitochondrion, theoretically; a small organelle, produces most of the energy in the form of ATP to supply the body. In Warburg's theory, the function of cellular mitochondrial respiration is dampened and mitochondria is not fully functional.

In the presence of Oxygen, most differentiated cells primarily metabolize glucose to carbon dioxide by oxidation of glycolytic pyruvate in the mitochondrial tricarboxylic cycle. In cancer cells including skin cancer cells, the metabolic shift is composed of increased glycolysis, activation of anabolic pathways including amino acid and pentose phosphate production, and increased fatty acid biosynthesis [5].

The explanations, so far, are only theories. Impaired cellular energy metabolism is the defining characteristic of nearly all cancers regardless of cellular or tissue origin [6]. In contrast to normal cells, which derive most of their usable energy from oxidative phosphorylation to meet energy demands. The Warburg effect, can be linked, theorically; to impaired mitochondrial function and energy metabolism.

However we must keep in mind that cell uses energy in many ways, so, when an energy failure exists, then there shall be a generalized failure, and the cancer cell is good example: alterations in growth signals, insensitivity to growth inhibitory signals, also apoptosis seems to disappear; the replicative potential appears limitless, increased angiogenesis, tissue invasion and metastasis, development of rapid resistance to chemo-therapy, etc.

Lactic acid is produced under aerobic and anaerobic glycolysis. Aerobic glycolysis can arise in tumor cells from damaged respiration whereas anaerobic glycolysis arises from the absence of oxygen. As oxygen will reduce anaerobic glycolysis and lactic acid production in most normal cells (Pasteur Effect); the continued production of lactic acid in the presence of oxygen can represent an abnormal Pasteur 
Effect. The replacement of respiration by fermentation (glycolysis) could be a symptom of cancer and not the cause [7]. Theories about genomic mutability selected in cancers cells, forgives that genetic code has not bad faith. In Nature there is no dogmas.

In hypoxic conditions the $\mathrm{NAD}^{+}$will essentially come via lactate dehydrogenase converting pyruvate into lactate. This reaction is prominent in tumor cells. Aerobic glycolysis in cancer cells involves elevated glucose uptake with lactic acid production in the presence of Oxygen. Genes for glycolysis are over expressed in the majority of cancer cells.

All cells require a constant level of useable energy to maintain viability. Intracellular levels of chemical energy are very strict, they must be within the same ranges were during all evolution, all of creation, it should not be neither more nor less. When these levels are inadequate, will destabilize the complex intracellular biochemical processes, and as the imbalance increases, it starts to grow, and while larger more rapid advances. Therefore the failure is widespread and seen instability in the genome, mutation, further respiratory impairment; apoptosis inhibition, etc. At the end, tumor progression.

To date, energy and respiration are considered synonyms. The current paradigm is that damage to cellular respiration, to mitochondria; and it precedes the power failure. There are several inconsistencies in the metabolism of cancer cells which are far from being able to be explained in the light of current theories. Under normal conditions, when glucose levels are low, the body mobilizes lipids and then muscle proteins to making glucose or ketones. But the cancer cell, despite having glucose and oxygen, anyway mobilize muscle protein and lipid stores.

Cancer cells rarely depend on mitochondria for respiration and obtain almost half ATP by directly metabolizing glucose to lactic acid. Thereby, bioenergetics may play a critical role in malignant transformation. Strikingly, embryonic stem cells and cancerous cells show a common concerted metabolic shift, including: enhanced glycolysis, decreased apoptosis and reduced mitochondrial respiration.

Since this cannot has been explained by way of the metabolism of glucose, he has tried to explain it by way of the levels of oxygen, and it is postulated that hypoxia may be a controller of functions in areas that could be considered hypoxic under normal situations. For example the regulation of functions of bone marrow hematopoietic cells and metastatic tumor cells, allegedly preserving primary functions important, fundamental; such as the control of the cell cycle, cell survival; metabolism; and protection against oxidative stress, although the latter even lacks definition.

Cancer cell has us so confused, that theories come and go. Refers to markers located on the cell surface, others posit the question that if cancer is a metabolic disease and that the genome instability is only a side effect; others have found that the cancer cell presents the Warburg effect and at the same time a reduced level of oxidative stress; others propose to turn to the glycolysis genes; and there are those who argue about the metastases, saying that the chemotherapy and radiation are the cause of these events than the intracellular changes that gave origin to the primary cancer. Furthermore, some are studying the biochemical bottlenecks that occur in the tumor cell, for example; Glycolysis is elevated in tumor cells, but the pyruvate kinase interrupts the conversion of the phosphoenolpyruvate to pyruvate. On the other hand, insulin or IGF, increases the flow of glucose within the cell, and therefore Glycolysis.
Cancer cells shows a broad spectrum of bioenergetic states, there is some evidence that metabolic coupling occur in aggressive tumors, between epithelial cancer cells and the stromal compartment; and between well-oxygenated and hypoxic compartments.

This ground breaking finding will changes basic concepts respect cell balance of mass and energy. These vicious cycles seems to be in our mind more than in cancer cell. Warburg's original work indicated that while glucose uptake and lactate production are greatly elevated, a cancer cell's rate of mitochondrial respiration is similar to that of normal cells. Warburg recognized that oxygen consumption is not diminished in tumor cells, but that respiration is altered because glycolysis persists in the presence of oxygen.

It is now clear that cancer cells utilize both glycolysis and oxidative phosphorylation to satisfy their metabolic needs. Experimental assessment of ATP production in cancer cells have demonstrated that oxidative pathways play a significant role in ATP generation; and may be responsible for about 50 to $80 \%$ of the ATP generated.

Cancer cells seem to mount an anti-oxidant defense and upregulate molecules that protect them against ROS and autophagy, preventing them from undergoing apoptosis. It seems that has been described in other diseases, for example chronic inflammatory diseases: that the cell returns to early stages of evolution.

The answer to the question of why cancer cells seem to prefer an inefficient metabolic pathway, glycolysis, it could give us the melanin. In our opinion, a cell that tends to Glycolysis is a cell that is experienced a regression in the evolution, i.e. tends to be more primitive. A healthy cell requires a very strict, very complex, order it cost, and is paid for with energy.

The glycolysis, rather than inefficient, is a primitive metabolic pathway that is closer to the bacterial cells to a human to them. And the behavior of cancer cells resembles the behavior of the bacteria in terms of organization. Metastases appear bacterial colonies. They do not have a complex order, not form systems, it is a regressive behavior. Eukaryote human cell has evolved based on the generation and distribution of chemical energy. And has been specializing more and more and forming more and more complex tissues. But the levels of chemical energy required to do so, must be the same that has taken over all the evolution, during all of creation. This cannot be changed, because everything revolves around these levels of chemical energy coming, now we know it; melanin.

\section{Implications in Cancer molecular biology}

We cannot heal what we don't understand. And the intrinsic property of melanin transform light energy into chemical energy through the water molecule dissociation, opens a huge panorama in the complicated current landscape of reactive medicine, that is, the medicine that acts after the disease appears.

Understanding the origin of life, by limiting the role of the glucose source of biomass and melanin as the source of energy of the human body, diseases will be studied in a very different way, as disease itself is merely a manifestation of an imbalance in the generation and distribution of energy. If this process basic, fundamental, is in the appropriate range, in the range that has had throughout all evolution, all of creation, the body will work as well as millions of years, has done millions of times.

A cancer cell is an expression of a living entity with low energy levels, as evidenced by the fact that the normal potential (110-120 
$\mathrm{mV}$ ) diminished to $70 \mathrm{mV}$. And action potentials also depend on the energy that emanates from the melanin. The cell uses energy in many ways, among them, to retain the shape. Therefore, when a body is deformed by the growth of a tumor, can be interpreted as that the body in question is not can keep shape, despite millions of years, have done million times; and is that the energy is needed to retain the shape.

Leaving behind the misconception that the glucose is the energy source, will allow us to significantly advance knowledge about molecular alterations in the cancer cell; in our opinion, the Warburg effect not has been able to be explained is because it is a reflection of widespread failure, what is typical of power, and that failures in any system.

This will allow us to implement a highly efficient preventive, predictive and personalized medicine, like never before.

\section{Conclusion}

Life cannot come spontaneously from the disorder. Life itself is a highly ordered sequence of chemical events which have evolved, adapting gradually over four billion years of evolution. We think that while more evolution, more specialization, so the use of energy is more and more efficient, more and more complex. A cell depends entirely on the generation and distribution of energy; as the entire universe. The order we see in living beings, starts from the source of energy that gave rise to it, in our case the melanin molecule.

The initial observation of Warburg in 1916 on tumor glycolysis with lactate production is still a crucial observation. And more than a point defect, Warburg's effect is a generalized failure, which is the typical behavior that follows an energy failure, and these in living and non-living systems. Maybe the Warburg effect can be interpreted as that the cancer cell is doing their best, given the conditions.

Metabolic cooperatively between adjacent cell-compartments is observed in several normal physiological settings. And with a common source of energy, in this case the melanin, rightly considered as the main source of energy of the cell, is then easier to understand how it happens.

\section{References}

1. Koolman J, Roehm KH (2005) Color Atlas of Biochemistry. Thieme, Stuttgart, New York. Second Edition.

2. Solís-Herrera A, Arias Esparza MC, Solís-Arias RI, Solís-Arias PE, Solís-Arias MP, et al. (2010) The unexpected capacity of melanin to dissociate the water molecule fills the gap between the life before and after ATP. Biomed Res 21: 224-227

3. Stefánsson E, Hatchell DL, Fisher BL, Sutherland FS, Machemer R (1986) Machemer, Robert. Panretinal Photocoagulation and Retinal Oxygenation in Normal and Diabetics Cats. Am J Ophthalmol 101: 657-664. [Crossref]

4. Solis-Herrera, Arturo. Solís-Arias, Martha Patricia (2013) The Odissey of Atmospheric Oxygen in their futile Attempt to reach the interior of the cell. Int $J$ Health Res and Innovation 1: 37-52.

5. Li W, Zhao Y (2012) Warburg Effect and Mitochondrial Metabolism in Skin Cancer, Epidermal Pigmentation, Nucleotide Excision Repair and Risk of Skin Cancer. $J$ Carcinogene Mutagene S4: 002.

6. Seyfred TN, Shelton LM (2010) Cancer as a Metabolic Disease. Nutr Metab 7: 7.

7. Israel M, Schwartz L (2011) The Metabolic Advantage of Tumor Cells. Mol Cancer: 10/70- 82. [Crossref]

Copyright: (C) 2015 Herrera AS. This is an open-access article distributed under the terms of the Creative Commons Attribution License, which permits unrestricted use, distribution, and reproduction in any medium, provided the original author and source are credited. 